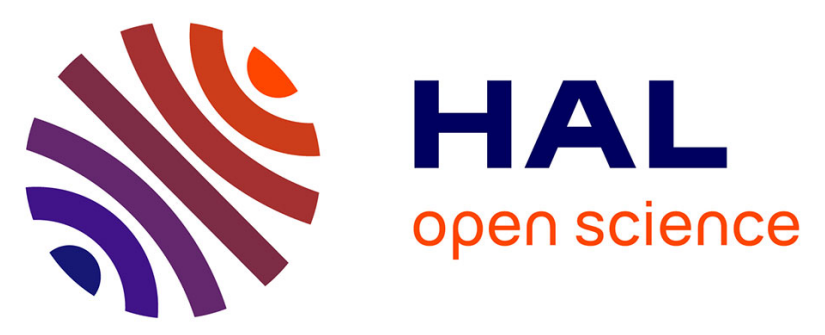

\title{
Influence of Linear Energy Transfer on the Nucleo-shuttling of the ATM Protein: A Novel Biological Interpretation Relevant for Particles and Radiation
}

Mira Maalouf, Adeline Granzotto, Clément Devic, Larry Bodgi, Melanie Ferlazzo, Christophe Peaucelle, Marcel Bajard, Jean-Yves Giraud, Jacques Balosso, Joël Hérault, et al.

\section{- To cite this version:}

Mira Maalouf, Adeline Granzotto, Clément Devic, Larry Bodgi, Melanie Ferlazzo, et al.. Influence of Linear Energy Transfer on the Nucleo-shuttling of the ATM Protein: A Novel Biological Interpretation Relevant for Particles and Radiation. International Journal of Radiation Oncology, Biology, Physics, 2019, 103 (3), pp.709-718. 10.1016/j.ijrobp.2018.10.011 . hal-02081379

\author{
HAL Id: hal-02081379 \\ https://hal.science/hal-02081379
}

Submitted on 21 Oct 2021

HAL is a multi-disciplinary open access archive for the deposit and dissemination of scientific research documents, whether they are published or not. The documents may come from teaching and research institutions in France or abroad, or from public or private research centers.
L'archive ouverte pluridisciplinaire HAL, est destinée au dépôt et à la diffusion de documents scientifiques de niveau recherche, publiés ou non, émanant des établissements d'enseignement et de recherche français ou étrangers, des laboratoires publics ou privés.

\section{(ㄷ)(1) $\$$}

Distributed under a Creative Commons Attribution - NonCommerciall 4.0 International 


\title{
Influence of Linear Energy Transfer on the Nucleo-shuttling of the ATM Protein: A Novel Biological Interpretation Relevant for Particles and Radiation
}

\author{
Mira Maalouf, PhD, ${ }^{* \dagger}$ Adeline Granzotto, BSc, ${ }^{*}$ Clément Devic, MSc, ${ }^{*}$ \\ Larry Bodgi, PhD, ${ }^{*}$, Mélanie Ferlazzo, PhD,* \\ Christophe Peaucelle, PhD, Marcel Bajard, PhD, \\ Jean-Yves Giraud, PhD, Jacques Balosso, MD, PhD, \\ Joël Hérault, PhD, "Marie-Claude Biston, PhD, " Claude Malet, PhD," \\ and Nicolas Foray, PhD*
}

${ }^{*}$ INSERM, UMR 1052, Radiobiology Group, Cancer Research Centre of Lyon, Lyon, France; ${ }^{\dagger}$ Lebanese University, Faculty of Sciences, Department of Chemistry and Biochemistry, Fanar, Beirut, Lebanon; ${ }^{\ddagger}$ American University of Beirut Medical Center, Department of Radiation Oncology, Riad El Solh, Ras Beirut, Beirut, Lebanon; ${ }^{\S}$ CNRS, Nuclear Physics Institute of Lyon, University Lyon 1, Villeurbanne, France; "University of Grenoble-Alpes, University Hospital of Grenoble, Radiation Oncology and Radiophysics Department, La Tronche, France; "Anti-cancer Center Antoine-Lacassagne, Nice, France; and ${ }^{\#}$ Anti-cancer Center Léon-Bérard, Lyon, France

Received Apr 9, 2018. Accepted for publication Oct 8, 2018.

\section{Summary}

The nucleo-shuttling of the ATM protein has recently been shown to influence the individual response to

\begin{abstract}
Purpose: Linear energy transfer (LET) plays an important role in radiation response. Recently, the radiation-induced nucleo-shuttling of ATM from cytoplasm to the nucleus was shown to be a major event of the radiation response that permits a normal DNA double-strand break (DSB) recognition and repair. Here, we aimed to verify the relevance of the ATM nucleo-shuttling model for high-LET particles and various radiation types.
\end{abstract}

Reprint requests to: Nicolas Foray, PhD, INSERM, UMR 1052, Groupe de Radiobiologie, Centre de Recherche en Cancérologie de Lyon, Bâtiment Cheney A, 28 Rue Laennec, 69008 Lyon, France. Tel: (33) 047878 28 28; E-mail: Nicolas.foray@inserm.fr

Mira Maalouf, Adeline Granzotto, and Clément Devic made equal contributions to this study.

Declaration of interest: WO 2015121597 A1, WO 2015121596 A1, WO 2017029449 A1. N.F. is scientific advisor of the Neolys Diagnostics start-up company.

Conflict of interest: None.

Supplementary material for this article can be found at https://doi.org/ 10.1016/j.ijrobp.2018.10.011.
Acknowledgments - The authors are thankful for the technical assistance of Christian Beaufrère, Jean-Pierre Lopez, Gersende Alphonse and Michael Beuve (Lyon University, Lyon, France), Isabelle Testard and JeanLouis Lefaix (GANIL, Caen, France), and Jean-Pierre Gérard and Jérôme Doyen (Antoine-Lacassagne anticancer Center, Nice, France). They thank the Association Pour la Recherche sur l'Ataxie-Telangiectasie (APRAT), the Electricité de France (Comité de Radioprotection), the Plan Cancer/ AVIESAN "Micromegas project," the Center National d'Etudes Spatiales (CNES), and the Commissariat Général à l'Investissement (INDIRA project). This work was supported by the Centre National d'Etudes Spatiales (CNES) and the Commissariat Général ă l'Investissement (Programmes d'Investissement d'Avenir - Projet INDIRA) 
radiation. However, the previous observations were performed with low linear energy transfer radiation. Here, the model of the nucleo-shuttling of the ATM protein is also relevant for high linear energy transfer particles, such as protons and carbon ions.
Methods and Materials: ATM- and H2AX-immunofluorescence was used to assess the number of recognized and unrepaired DSB in quiescent fibroblast cell lines exposed to x-rays, $\gamma$-rays, 9 - and $12-\mathrm{MeV}$ electrons, 3 - and $65-\mathrm{MeV}$ protons and 75-MeV/u carbon ions.

Results: The rate of radiation-induced ATM nucleo-shuttling was found to be specific to each radiation type tested. By increasing the permeability of the nuclear membrane with statin and bisphosphonates, 2 fibroblast cell lines exposed to high-LET particles were shown to be protected by an accelerated ATM nucleo-shuttling.

Conclusions: Our findings are in agreement with the conclusion that LET and the radiation/particle type influence the formation of ATM monomers in cytoplasm that are required for DSB recognition. A striking analogy was established between the DSB repair kinetics of radioresistant cells exposed to high-LET particles and that of several radiosensitive cells exposed to low-LET radiation. Our data show that the nucleo-shuttling of ATM provides crucial elements to predict radiation response in human quiescent cells, whatever the LET value and their radiosensitivity. (C) 2018 Elsevier Inc. All rights reserved.

\section{Introduction}

There is a large body of evidence that each type of ionizing radiation (IR) and particles induces a specific spectrum of energy microdepositions differing by their frequency, energy density, and spatial distribution. ${ }^{1-5}$ IR and particles produce 3 major types of DNA damage: base damage (BD), DNA single-strand breaks (SSB), and DNA double-strand breaks (DSB). Because energy microdepositions of more than 1,10 , and $100 \mathrm{eV} / \mathrm{nm}^{3}$ are required for the occurrence of $\mathrm{BD}, \mathrm{SSB}$, and DSB, respectively, the physical features of IR and particles condition the induction rates of BD, SSB, and DSB. For example, 1-Gy $\gamma$-rays simultaneously produces approximately 10,000 BD, 1000 SSB and 40 DSB per human diploid cells, whereas the same dose of $\alpha$ particles produces less BD and more DSB. ${ }^{4,6-8}$

The energy microdepositions also condition the DNA damage reparability: the denser the energy microdeposition, the less repairable the resulting DNA damage. For example, in human radioresistant cells, $50 \%$ of BD are repaired in 5 to 10 minutes, whereas the same proportion of DSB requires 50 to 60 minutes. ${ }^{4,6,7}$ Furthermore, DSB induced by high-linear energy transfer (LET) particles may be less frequent but also less repairable than those induced by low-LET ones. ${ }^{4,6,7}$ The current paradigm to explain the relative biological efficiency (RBE) of high-LET radiation and particles is that dense energy microdepositions produce more irreparable DNA damage. ${ }^{4,6,7,9}$ Although there is a large body of evidence that unrepaired DSBs are correlated with radiosensitivity, ${ }^{10,11}$ the DSB endpoints predictive of radiosensitivity remain to be clarified.

Since 2003, hundreds of skin fibroblasts deriving from patients suffering from genetic diseases linked to radiosensitivity or showing abnormal tissue reactions after radiation therapy were collected. ${ }^{12}$ From this collection, the ATM protein, a major actor of the IR response has been suggested to be a cytoplasmic protein that translocates in the nucleus after irradiation. The presence of ATM monomers in the nucleus has been shown to ensure the phosphorylation of $\mathrm{H} 2 \mathrm{AX}$ histone variants $(\gamma \mathrm{H} 2 \mathrm{AX})$, an early sensor of the recognition of DSB by the nonhomologous end-joining (NHEJ) pathway. The ATM nucleoshuttling, quantified by the number of nuclear foci formed by the auto-transphosphorylation forms of ATM (pATM), was shown to be the basis of powerful predictive assays. ${ }^{12-18}$ This model is consistent with the findings that ATM monomers are more active than ATM dimers. ${ }^{19,20}$ Furthermore, the ATM nucleo-shuttling model provides a novel biological interpretation of the linear-quadratic formula that is also consistent with the hypersensitivity to the low-dose phenomenon. ${ }^{14}$

Here, by applying $\gamma \mathrm{H} 2 \mathrm{AX}$ and pATM immunofluorescence assays to some human quiescent fibroblasts from different origin and exposed to 6-MV X-rays, $\gamma$-rays, 9- and $12-\mathrm{MeV}$ electrons, 3- and $64-\mathrm{MeV}$ protons, and $75-\mathrm{MeV} / \mathrm{u}$ carbon ions, we verified whether the model of the ATM nucleo-shuttling is relevant whatever the LET and the radiation or particle type.

\section{Methods and Materials}

\section{Cell lines}

The 149BR, 1BR3, and AG1521 untransformed human fibroblast cell lines were chosen for their genomic stability and the abundant literature associated with their radioresistance. ${ }^{12,21,22}$ The radiobiological characteristics of the ATM-mutated AT4BI and AT5BI and the LIG4-mutated 180BR hyper-radiosensitive fibroblast cell lines were published elsewhere. ${ }^{21,22}$ The radiobiological characteristics of the 01HNG and 06CLB fibroblast cell lines derived from patients showing severe tissue reactions after radiation therapy were published elsewhere. ${ }^{12}$ All the experiments were performed with quiescent fibroblasts. Cell culture conditions are detailed in supplementary data (SD). ${ }^{21}$ 


\section{Statin and bisphosphonate treatment}

The zoledronate plus pravastatin (ZOPRA) treatment was applied as published previously. ${ }^{23}$ Cells were incubated with $1 \mu \mathrm{M}$ pravastatin (Sigma-Aldrich France, SaintQuentin-Fallavier, France) for 24 hours. Thereafter, $1 \mu \mathrm{M}$ zoledronate (Sigma-Aldrich) was added into the culture medium and cells were incubated for 12 hours. The culture medium was renewed immediately before irradiation.

\section{Immunofluorescence}

Immunofluorescence protocol and foci scoring were described elsewhere ${ }^{12,24}$ and detailed in SD. Anti$\gamma H 2 A X^{\text {ser139 }}$ (\#JBW301) and -pATM ${ }^{\text {ser1981 }}$ (\#10H11.E12) antibodies were applied at 1:800 and 1:100, respectively (Millipore, Darmstadt, Germany).

\section{Low-LET irradiation}

Dosimetry certifications and the irradiations were performed with a 6 MV X-rays clinical irradiator (SL 15 Phillips; doserate, $4 \mathrm{~Gy} / \mathrm{min}$ ) at anti-cancer Centre Léon-Bérard, (Lyon, France $){ }^{16}$ with ${ }^{60} \mathrm{Co}(1.25 \mathrm{MeV})$ and ${ }^{137} \mathrm{Cs}(0.662 \mathrm{MeV})$ g-rays sources (dose-rate, 2 and $4 \mathrm{~Gy} / \mathrm{min}$, respectively) at anti-cancer Centre Gustave-Roussy (Villejuif, France) ${ }^{21,25}$ and with an orthovoltage $250 \mathrm{kV}$ X-rays Phillips irradiator (dose-rate, $4 \mathrm{~Gy} / \mathrm{min}$ ) at the European Synchrotron Radiation Facility (Grenoble, France) ${ }^{26}$ (Table E1; available online at https://doi.org/10.1016/j.ijrobp.2018.10.011).

\section{Electrons irradiation}

Dosimetry certifications and electrons irradiations were performed at 9 and $12 \mathrm{MeV}$ (dose-rate, $4 \mathrm{~Gy} / \mathrm{min}$ ) with a Clinac Varian accelerator at Grenoble University Hospital (Grenoble, France) (Table E1).

\section{Protons irradiation}

Irradiation with the $3 \mathrm{MeV}$ protons was performed with the 4-MV Van de Graaff accelerator of University of Lyon (Lyon, France) (dose-rate $>10 \mathrm{~Gy} / \mathrm{min}$ ) (Table E1). The $65-\mathrm{MeV}$ protons irradiations were performed with the MEDICYC medical proton therapy cyclotron of the anticancer Center of Nice (France) (dose-rate: $2 \mathrm{~Gy} / \mathrm{min}$ ) and the physical features and dosimetry were published elsewhere $^{25-27}$ (Table E1). ${ }^{27-29}$

\section{Carbon ions irradiation}

The carbon ions irradiations were performed at the Grand Accelerateur National d'Ions lourds (GANIL, Caen, France) (dose-rate, $2 \mathrm{~Gy} / \mathrm{min}$ ). The carbon ions were delivered at $75 \mathrm{MeV}$ per nucleon ( $72 \mathrm{MeV} / \mathrm{u}$ in cells). The physical features and dosimetry were published elsewhere (Table E1). ${ }^{30-32}$

\section{Statistical analysis}

For practical reasons, the maximal dose used with carbon ions was $1 \mathrm{~Gy}$. For all the other particle and radiation types, the dose applied was 2 Gy. To permit relevant data comparisons, foci data were expressed, when indicated, as a percentage of the $\gamma$-rays reference data obtained at $10 \mathrm{mi}$ nutes after irradiation (ie, $80 \mathrm{DSB} / \mathrm{Gy} /$ cell). This data transformation does not generate biases because the DSB repair rate of radioresistant cells when expressed as percentage of unrepaired DSB was shown to be independent of the dose and the technique used. ${ }^{33}$ The fitting formula for DSB repair kinetics ${ }^{34}$ and the RBE calculations are described in SD.

\section{Results}

The assessment of $\gamma \mathrm{H} 2 \mathrm{AX}$ foci in cells exposed to low-LET radiation

The kinetics of the appearance and disappearance of $\gamma H 2 A X$ foci are characterized by 2 phases: the foci appearance phase, which reflects the DSB recognition step, and the foci disappearance phase, which is interpreted as the DSB repair step. ${ }^{34}$ By using pulsed-field gel electrophoresis or $\gamma \mathrm{H} 2 \mathrm{AX}$ immunofluorescence, exposure of the 149BR, 1BR3, and AG1521 radioresistant fibroblasts to $250-\mathrm{kV}$ or 6-MV X-rays and ${ }^{60} \mathrm{Co}$ or ${ }^{137} \mathrm{Cs} \gamma$-rays leads to a DSB induction rate of $37 \pm 4$ DSB per gray per cell, whatever the precited radiation types, in agreement with other published data. ${ }^{4,21,25}$ The maximal number of $\gamma \mathrm{H} 2 \mathrm{AX}$ foci was reached at 10 minutes after irradiation after $2 \mathrm{~Gy}$. At more than 24 hours after, negligible yields of residual $\gamma \mathrm{H} 2 \mathrm{AX}$ foci were observed in the 3 cell lines, which was in agreement with previously published data (Fig. 1A; Table E2; available online at https://doi.org/10. 1016/j.ijrobp.2018.10.011). ${ }^{6,12,21,22,25,35}$

\section{Assessment of $\gamma \mathrm{H} 2 \mathrm{AX}$ foci in cells exposed to electrons}

Ten minutes after irradiation with $9-\mathrm{MeV}$ electrons, the percentages of $\gamma \mathrm{H} 2 \mathrm{AX}$ foci assessed in the 149BR, 1BR3, and AG1521 radioresistant cell lines were found to be similar $(85 \% \pm 4 \%, 82 \% \pm 4 \%$, and $88 \% \pm 2 \%$, respectively; $P>.8$ ) but significantly different from the corresponding X-rays data $(P<.01)$. Twenty-four hours after irradiation, the numbers of residual $\gamma \mathrm{H} 2 \mathrm{AX}$ foci were found significantly higher with electrons $(4 \% \pm 1 \%$, $4 \% \pm 1 \%$, and $3 \% \pm 1 \%$, respectively; $P<.05$ ) than with $\mathrm{X}$-rays, suggesting that DSB produced by $9 \mathrm{MeV}$ electrons are less repairable than those induced by X-rays (Fig. 1A; Table E2). Similar conclusions were reached at $12 \mathrm{MeV}$ $(4 \% \pm 1 \%, 5 \% \pm 1 \%$, and $5 \% \pm 1 \%$, respectively; $P<.05)$. 

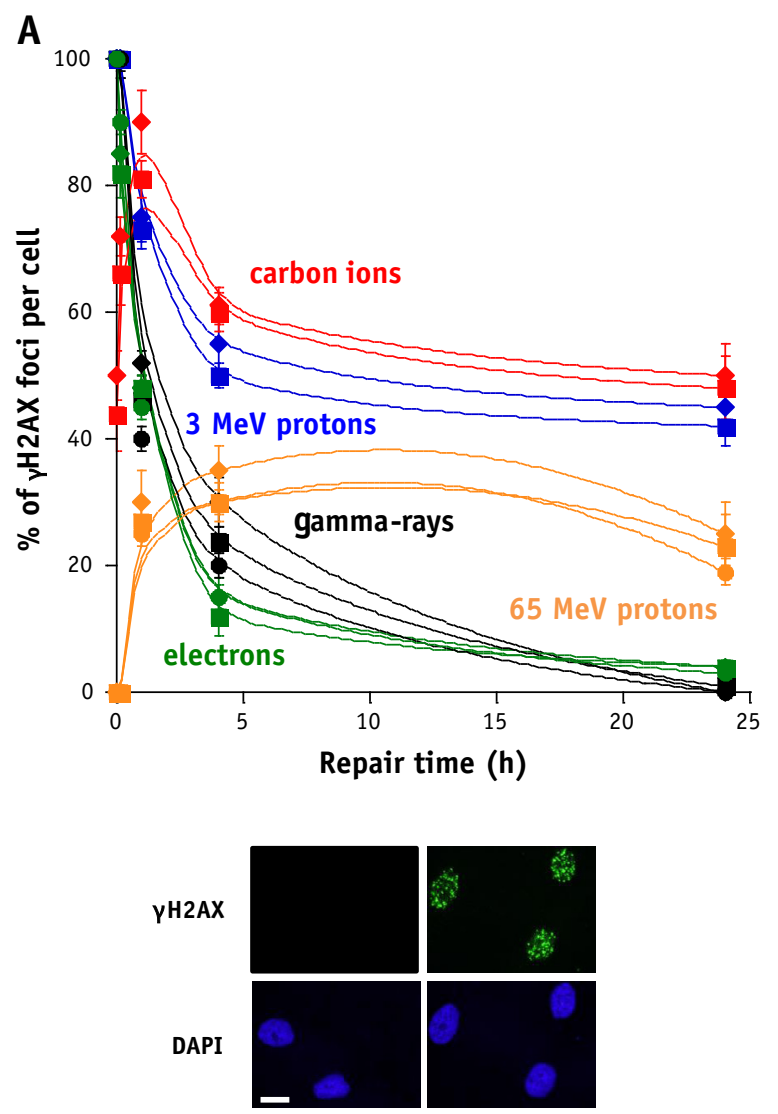
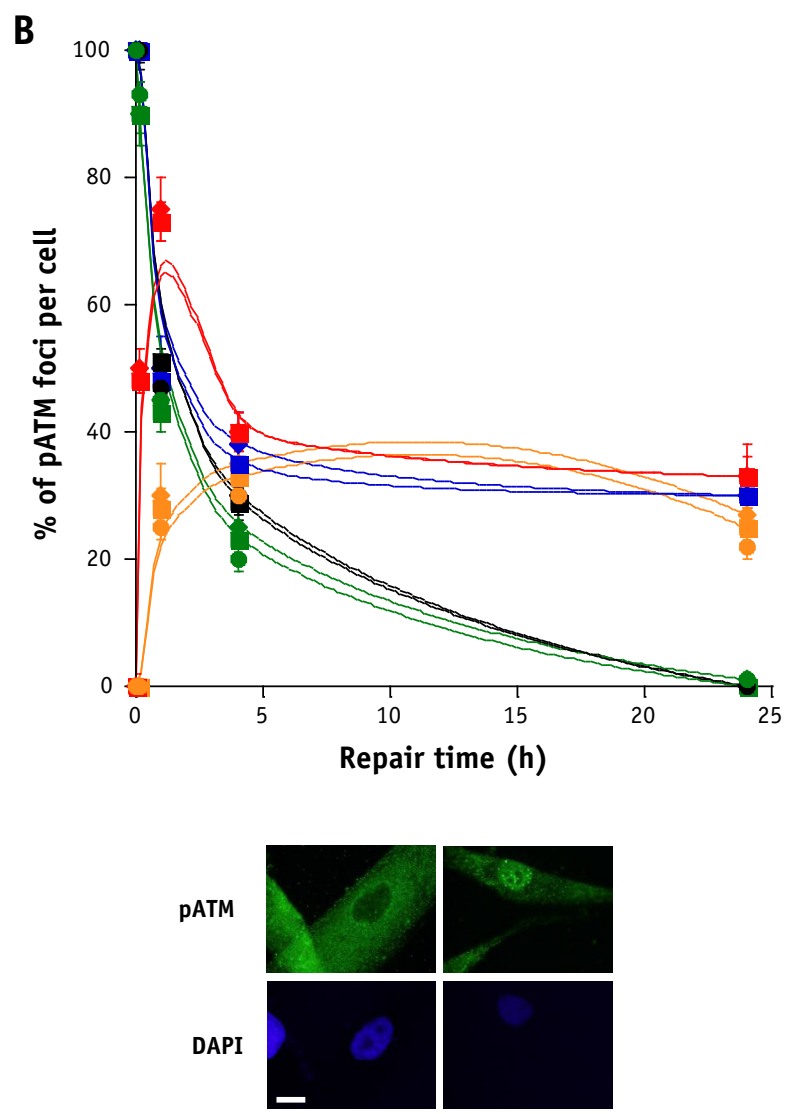

Fig. 1. Kinetics of $\gamma \mathrm{H} 2 \mathrm{AX}$ and $\mathrm{pATM}$ foci with different radiation types. Immunofluorescence against (A) $\gamma \mathrm{H} 2 \mathrm{AX}$ and (B) pATM was applied to the radioresistant 149BR (diamonds), 1BR3 (squares), and AG1521 (circles) fibroblast cell lines. Data are represented as a percentage of the number of foci assessed 10 minutes after irradiation with $\gamma$-rays. Each plot represents the mean \pm standard error of the mean of triplicates. The lower panels show representative immunofluorescence images (149BR cells, 3-MeV protons, 0 and 4 hours after irradiation) with the indicated biomarkers. White bars represent $10 \mu \mathrm{m}$.

\section{Assessment of $\gamma \mathrm{H} 2 \mathrm{AX}$ foci in cells exposed to protons}

Ten minutes after irradiation with $3 \mathrm{MeV}$ protons, the percentages of $\gamma \mathrm{H} 2 \mathrm{AX}$ foci assessed in the 149BR and 1BR3 fibroblast cell lines $(100 \% \pm 0 \%$ for both) were found to be similar to that obtained with X-rays. By contrast, 10 minutes after irradiation with $65-\mathrm{MeV}$ protons, the percentages of $\gamma \mathrm{H} 2 \mathrm{AX}$ foci assessed in the 149BR, 1BR3, and AG1521 fibroblast cell lines were found negligible $(0 \pm 0 \%$ for all the cell lines).

These findings suggest that DSB recognition is impaired with $65 \mathrm{MeV}$ protons but normal with $3-\mathrm{MeV}$ protons. In the same cell lines tested, the percentages of $\gamma \mathrm{H} 2 \mathrm{AX}$ foci assessed with $3-\mathrm{MeV}$ protons $(45 \% \pm 3 \%$ and $42 \% \pm 3 \%$, respectively) were found to be clearly higher than with $\mathrm{X}$-rays. A similar conclusion was reached with $65-\mathrm{MeV}$ protons $(25 \% \pm 5 \%, 23 \% \pm 5 \%$, and $19 \% \pm 2 \%$, respectively), although the $3-\mathrm{MeV}$ protons data were higher than the $65-\mathrm{MeV}$ protons ones. Altogether, these data suggest that DSB produced by protons are less repairable than those produced by $\mathrm{X}$-rays, in agreement with the literature (Fig. 1A; Table E2). ${ }^{36,37}$

\section{Assessment of $\gamma \mathrm{H} 2 \mathrm{AX}$ foci in cells exposed to carbon ions}

Ten minutes after irradiation, the percentages of $\gamma \mathrm{H} 2 \mathrm{AX}$ foci in the 149BR and 1BR3 fibroblast cell lines were found to be significantly lower with carbon ions $(72 \% \pm 3 \%$ and $66 \% \pm 6 \%$, respectively; $P>.8$ ) than with $\mathrm{X}$-rays $(P<.001)$. Furthermore, these percentages reached its maximum at 1 hour $(90 \% \pm 3 \%$ and $81 \% \pm 5 \%$, respectively). The percentages of residual $\gamma \mathrm{H} 2 \mathrm{AX}$ foci were found to be clearly higher than with X-rays $(50 \% \pm 5 \%$ and $48 \% \pm 5 \%$, respectively). The DSB induced by carbon ions therefore appeared weakly recognized by NHEJ and slowly repairable, as suggested elsewhere (Fig. 1A; Table E2). ${ }^{31}$

\section{Assessment of pATM foci in cells exposed to various types of radiation}

With $65-\mathrm{MeV}$ protons and carbon ions, the percentage of $\gamma \mathrm{H} 2 \mathrm{AX}$ foci at 10 minutes after irradiation was found lower than that assessed with X-rays and the maximal percentage of $\gamma \mathrm{H} 2 \mathrm{AX}$ foci was not reached at 10 minutes but at 1 hour. The 

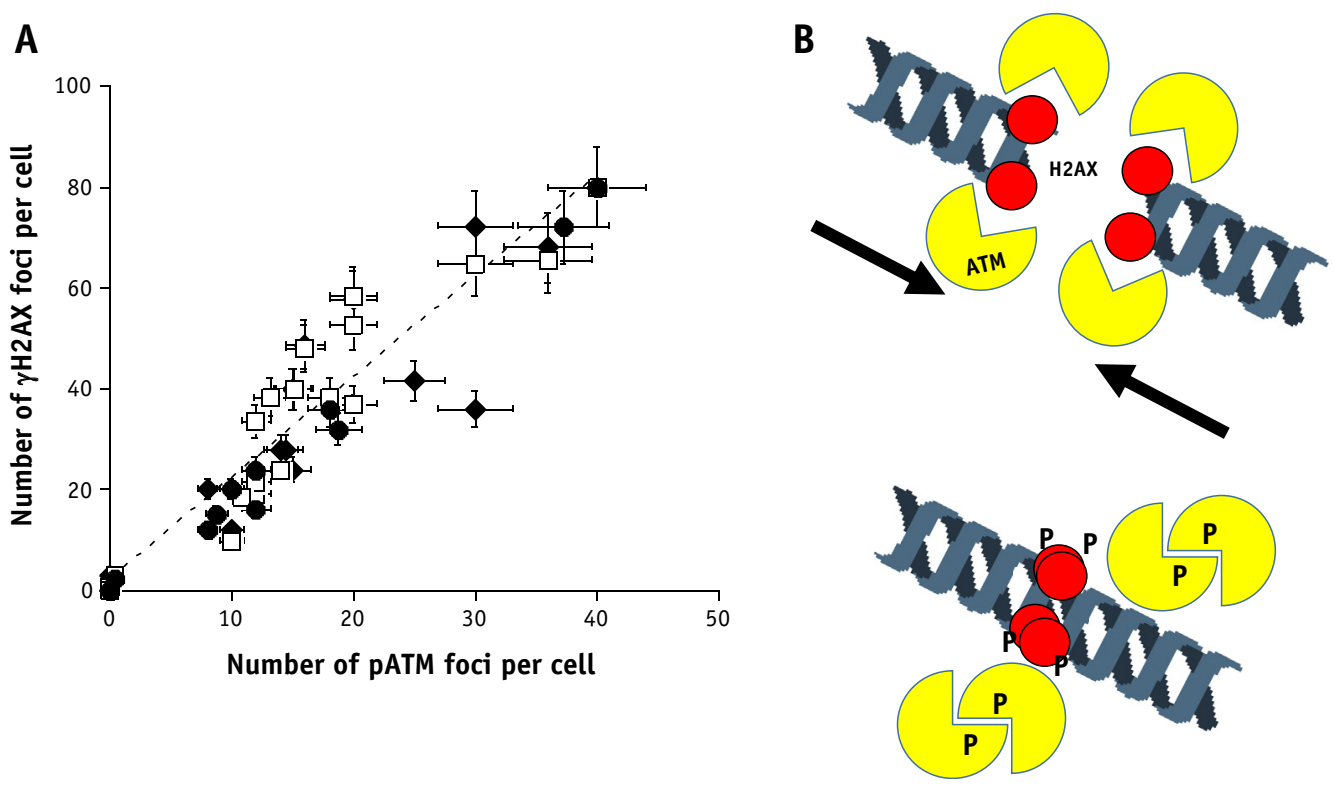

Fig. 2. Relationship between $\gamma \mathrm{H} 2 \mathrm{AX}$ and pATM foci. (A) The numbers of $\gamma \mathrm{H} 2 \mathrm{AX}$ foci obtained with different radiation types shown in Fig. 1 were plotted against the numbers of pATM foci corresponding to the same postirradiation time (data shown in Fig. 1). A significant linear regression relationship was observed for each cell lines (149BR, diamonds: $y=2.62+1.89 x, r=0.93, P<.01$; 1BR3, squares: $y=2.79+1.99 x, r=0.94, P<.01$; AG1521, circles: $y=-1.32+1.97 x, r=0.99, P<.001$ ). (B) Schematic illustration of the phosphorylation of $\gamma \mathrm{H} 2 \mathrm{AX}$ molecules by ATM monomers. The DNA end-joining results in a lower distance between ATM monomers of each end, which should facilitate the ATM redimerization and the formation of nuclear pATM foci.
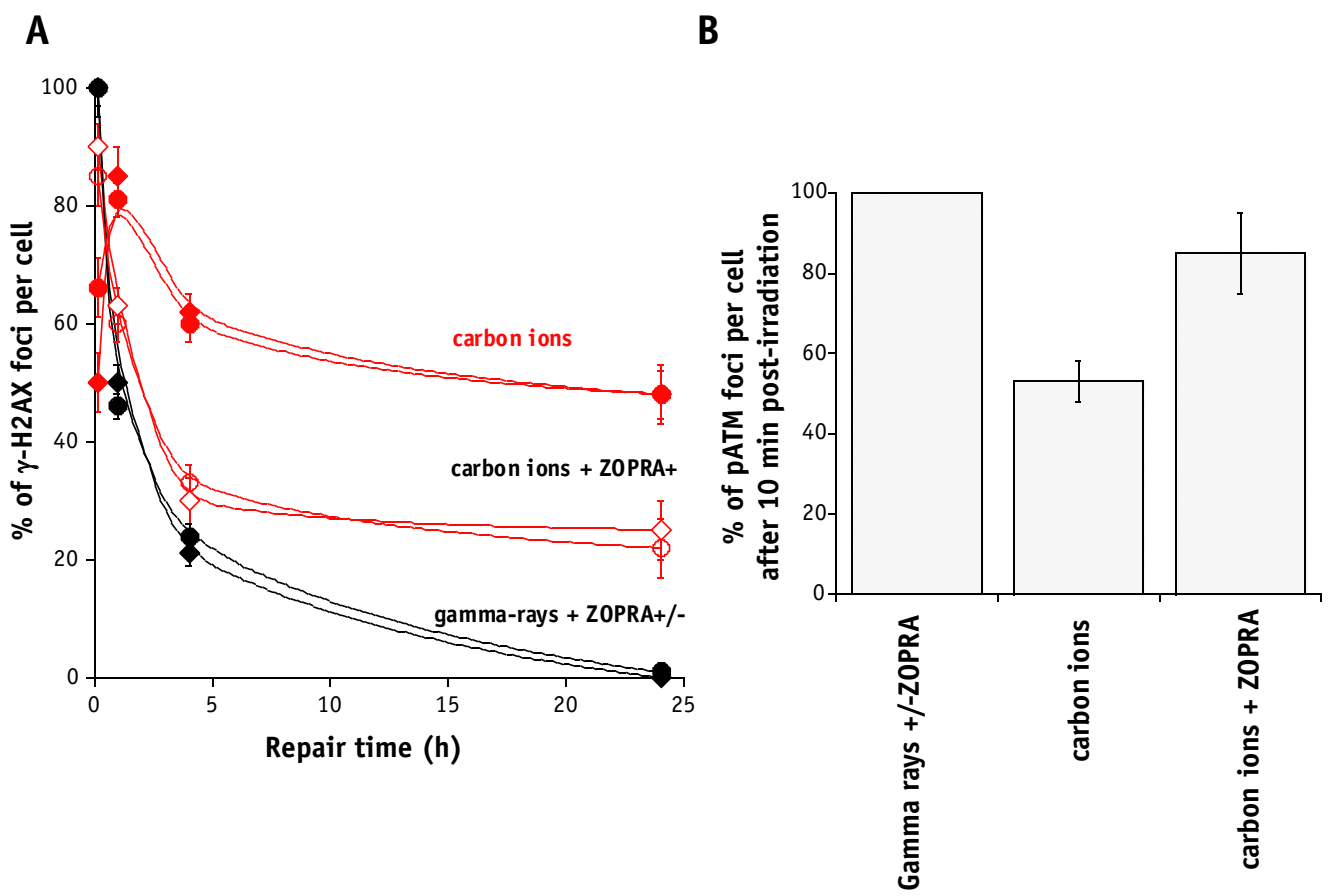

Fig. 3. Kinetics of $\gamma \mathrm{H} 2 \mathrm{AX}$ and pATM foci after exposure to carbon ions and ZOPRA treatment. (A) Immunofluorescence against $\gamma \mathrm{H} 2 \mathrm{AX}$ foci was applied to the radioresistant 149BR (diamonds) and AG1521 (circles) fibroblast cell lines presubmitted to ZOPRA treatment (open symbols) or not (closed symbols) and irradiated with carbon ions (1 Gy). Dotted lines and corresponding plots represent the data obtained with or without ZOPRA treatment followed by $\gamma$-ray irradiation. (B) The corresponding pATM data in 149BR cells obtained 10 minutes after irradiation were plotted at the indicated treatments. Each plot represents the mean \pm standard error of the mean of duplicates. 
pattern of this kinetics is not unusual, because it was already observed in some radiosensitive cells, ${ }^{38,39}$ suggesting a lack of an early DSB recognition and a delayed ATM nucleoshuttling. We therefore applied antipATM immunofluorescence in the same conditions as described earlier. With X-rays, the radioresistant 149BR, 1BR3, and AG1521 cells showed similar numbers of pATM foci per gray per cell at 10 minutes after irradiation $(37 \% \pm 3 \%, 38 \% \pm 5 \%$, and $36 \% \pm 6 \%$, respectively; $P>.8$ ). The percentages of pATM foci decreased to zero at 24 hours after irradiation for the 3 cell lines tested (Fig. 1B). These findings fully agreed with other data published elsewhere. ${ }^{12,16}$ With electrons, the percentages of pATM foci were not found different from that assessed with $\mathrm{X}$-rays. With $3-\mathrm{MeV}$ protons, the pATM data of the 2 cell lines tested (149BR and 1BR3) are in agreement with the corresponding $\gamma \mathrm{H} 2 \mathrm{AX}$ data with a normal pATM foci value for the first hour following irradiation and a slower disappearance rate of pATM foci after this time. With carbon ions, for both cell lines, the percentage of pATM foci past 10 minutes after irradiation was significantly lower than that observed after Xray irradiation. However, the percentages of pATM foci induced by carbon ions past 10 minutes after irradiation were found clearly lower that those obtained with X-rays. Past 24 hours after irradiation, the percentages of the residual pATM foci with 3- and 65-MeV protons and with carbon ions were found systematically higher than those observed after Xrays in the indicated cell lines (Fig. 1B).

As specified earlier, the phosphorylation of $\gamma \mathrm{H} 2 \mathrm{AX}$ histone triggers DSB repair via NHEJ pathway. The DNAend-joining results in the reduction of the distance between ATM monomers situated on each end, which facilitates the redimerization of ATM and, consequently, the formation of nuclear pATM foci. ${ }^{12}$ Hence, the number of $\gamma \mathrm{H} 2 \mathrm{AX}$ should be 2-fold larger than that of pATM foci. By plotting the number of $\gamma \mathrm{H} 2 \mathrm{AX}$ foci assessed in the 149BR, 1BR3, and AG1521 cell lines for a given time after irradiation against the corresponding number of pATM foci, we observed a ratio that was not statistically different from 2 , in agreement with the prior assumption (Fig. 2).

\section{Effect of statins and bisphosphonates on cells irradiated with carbon ions}

The ZOPRA treatment was previously shown to accelerate the nucleo-shuttling of ATM. ${ }^{23}$ Because the percentages of $\gamma \mathrm{H} 2 \mathrm{AX}$ and pATM foci per cell past 10 minutes after irradiation were found to be significantly lower with carbon ions than with X-rays, the effect of the ZOPRA treatment was investigated in the 149BR and 1BR3 radioresistant cell lines exposed to carbon ions. Interestingly, the ZOPRAtreated cells elicited a larger number of $\gamma \mathrm{H} 2 \mathrm{AX}$ foci than untreated cells, suggesting that the low number of ATM monomers and their weak diffusion in the nucleus observed with carbon ions can be partially corrected by the ZOPRA treatment via the acceleration of the nucleo-shuttling of ATM (Fig. 3A). This hypothesis was supported by pATM data (Fig. 3B). It is noteworthy that ZOPRA-treated 149BR and 1BR3 cells exposed to $\gamma$-rays did show similar kinetics of $\gamma \mathrm{H} 2 \mathrm{AX}$ foci to untreated cells. ${ }^{16}$

\section{Discussion}

\section{Model of the ATM nucleo-shuttling is relevant for high-LET particles and radiation}

According to the recent resolution of the linear-quadratic model published elsewhere, ${ }^{14} 2$ categories of unrepairable DSB can be lethal:

- The $\alpha$-type DSB, recognized by NHEJ but remaining unrepaired after 24 hours. These DSBs are reflected by early pATM foci but persistent residual $\gamma-\mathrm{H} 2 \mathrm{AX}$ foci.
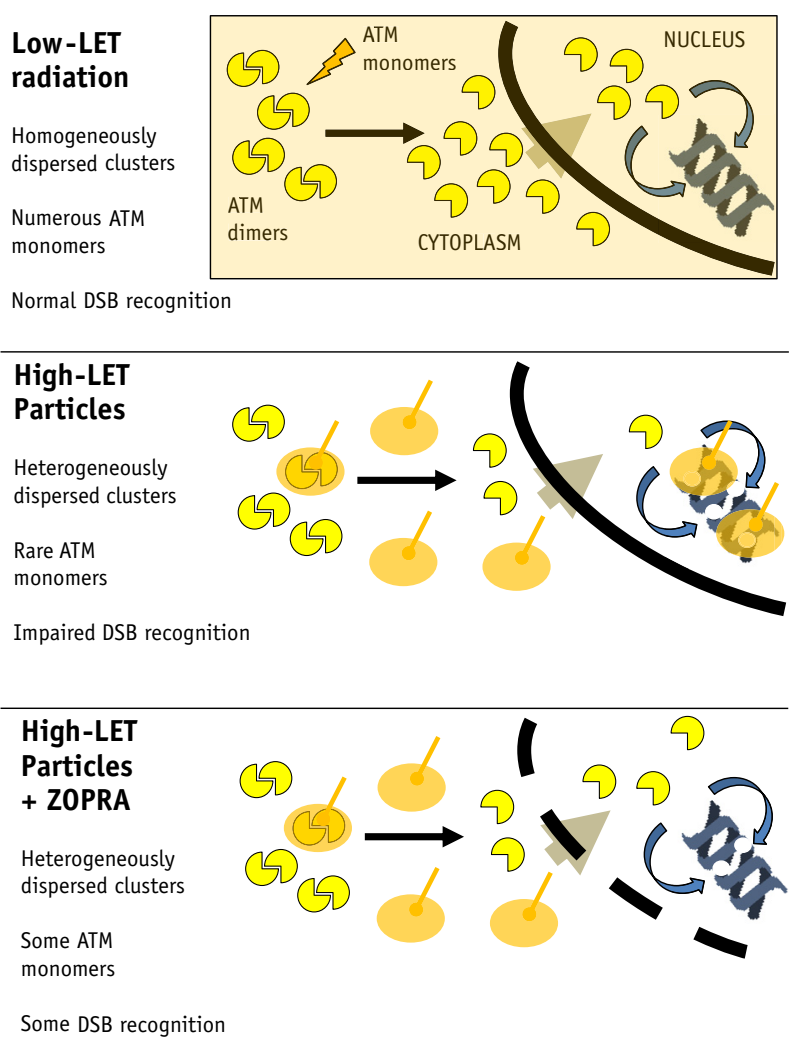

Fig. 4. Recapitulative scheme of the effect of the ATM nucleo-shuttling of ATM in the response to high-LET particles. Low-LET radiation induces a homogeneous oxidation that permits the formation of numerous ATM monomers. They rapidly diffuse into the nucleus, thus contributing to DSB recognition and repair. After exposure to high-LET particles, the ionization clusters are not sufficiently dispersed to interact with ATM dimers to produce numerous ATM monomers. DSB recognition is impaired. If ZOPRA treatment is applied, the permeability of the nuclear membranes is increased, and some additional ATM monomers can diffuse into the nucleus to participate to the DSB recognition and repair. 

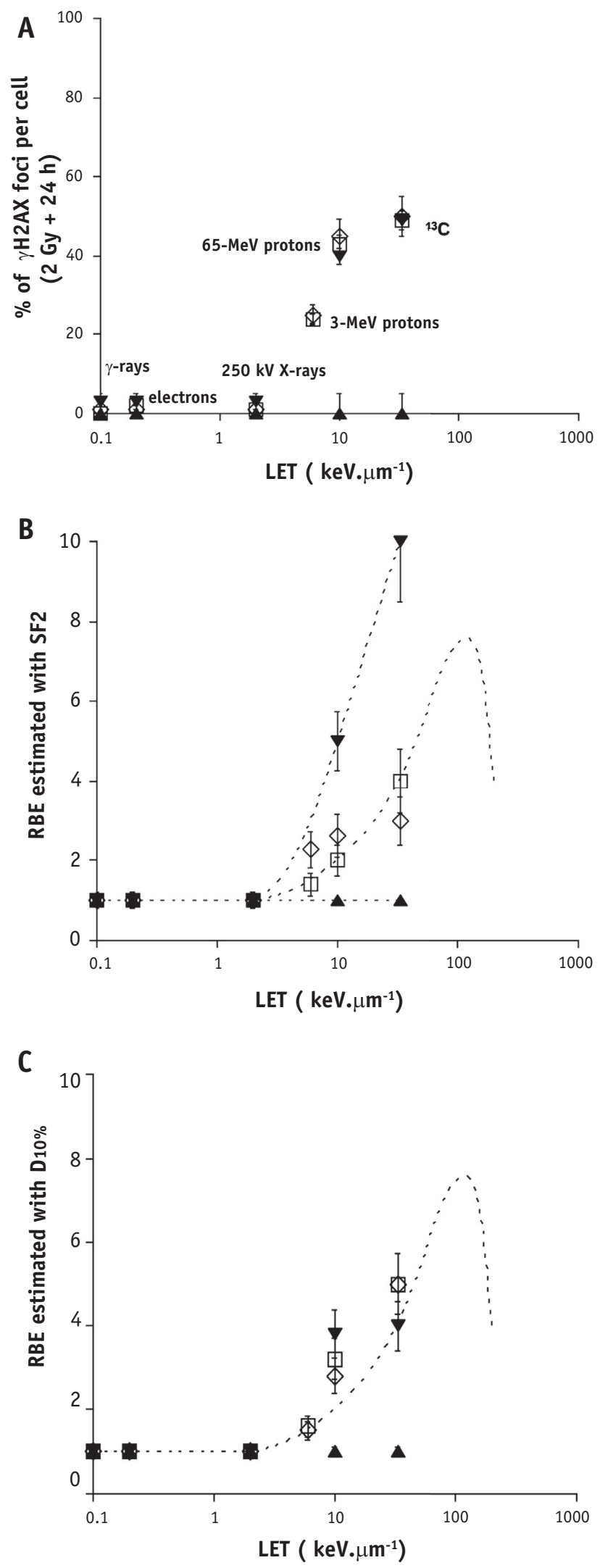

Fig. 5. RBE-LET relationships. (A) The number of residual $\gamma$-H2AX foci shown in Fig. 1A (the $\gamma$-rays data were taken as a reference) were plotted against the LET values corresponding to each radiation type for the radioresistant
- The $\beta$-type DSB not recognized by any DSB repair pathways and therefore never repaired. These DSB are not directly visible by pATM and $\gamma$-H2AX immunofluorescence, and they correspond to a low number of pATM foci.

In the case of $3 \mathrm{MeV}$ protons, the energy microdepositions distribution is likely to be quantitatively and qualitatively sufficient to produce a minimal flux of ATM monomers to recognize all the DSBs produced in the nucleus. Conversely, the energy microdeposition might be so dense that the DSBs created are unrepairable; therefore, the $\alpha$-type DSB contribution might be predominant for $3 \mathrm{MeV}$ protons. Hence, exposure to $3-\mathrm{MeV}$ protons can result in severe DSB but fully recognized by NHEJ, in agreement with our data (Fig. 4).

In the case of $65-\mathrm{MeV}$ protons, the energy microdepositions can be so scattered that the production of ATM monomers would not be sufficiently high to recognize all the induced DSBs. Conversely, their energy density might be sufficiently high to produce unrepairable DSB like with 3-MeV protons; therefore, the $\beta$-type DSB contribution might be predominant for $65-\mathrm{MeV}$ proton. Hence, exposure to $65-\mathrm{MeV}$ protons can result in severe DSB but poorly recognized by NHEJ, in agreement with our data (Fig. 4).

In the case of carbon ions, the scenario is intermediate between that of 65- and 3-MeV protons: the flux of ATM monomers might be significant but still not sufficiently high to recognize all the DSBs at 10 minutes after irradiation. Hence, both $\alpha$ - and $\beta$-type DSBs contribute to the RBE of carbon ions (Fig. 4).

In the case of 9- to $12-\mathrm{MeV}$ electrons, X-rays, and $\gamma$-rays, the LET is low and the energy microdepositions are distributed more homogeneously in cells. Consequently, the flux of ATM monomers might be higher than with highLET particles. As a result, all the DSBs are generally recognized, and their reparability is high, probably because of the energy microdepositions that are less dense than with high-LET particles (Fig. 4).

The effect of the nucleo-shuttling of ATM after exposure to high-LET particles was also illustrated by the radioprotective effect of the ZOPRA treatment shown in Fig. 3. Because the ZOPRA treatment acts on the permeability of the nuclear membranes, it demonstrates that a

149BR (diamonds), 1BR3 (squares), moderately radiosensitive (group II) 01HNG (inverted triangles), and hyperradiosensitive (group III) AT5BI (triangles) fibroblast cell lines. For each radiation type, using previously published formulas, ${ }^{6,28}$ we calculated (B) SF2 and (C) $D_{10 \%}$ from the $\gamma$-H2AX and pATM data, respectively. The RBE values were deduced thereafter according to the methods detailed in supplementary data. The RBE values were plotted against the corresponding LET. The dotted line represents the RBE-LET curve shown by Steel. ${ }^{35}$ 
facilitated-accelerated diffusion of ATM monomers can be sufficient for improving the recognition of DSB by NHEJ and therefore for stimulating DSB repair (Fig. 4). Further study is needed to investigate whether ZOPRA can serve as a radioprotection approach for healthy tissues without modulating the antitumor efficiency.

\section{RBE versus LET}

Because the number of unrepaired DSBs was shown to be correlated with cell survival, we plotted the RBE calculated from the numbers of residual $\gamma-\mathrm{H} 2 \mathrm{AX}$ foci shown in Fig. 1A against the LET values corresponding to each radiation type (Table E1; Fig. 5A). Using previously published formulas, ${ }^{14,22}$ we also calculated the corresponding cell surviving fraction at $2 \mathrm{~Gy}$ (SF2) and the dose reached when cell surviving fraction was equal to $10 \%\left(\mathrm{D}_{10 \%}\right)$ from the $\gamma \mathrm{H} 2 \mathrm{AX}$ and pATM data, respectively. The RBE values were deduced thereafter (Fig. 5B, 5C; SD). The resulting RBE-LET curves of 2 radioresistant fibroblast cell lines tested (149BR and 1BR3) were in good agreement with the literature data, even if the RBE values strongly depend on the calculation method. ${ }^{8}$ However, the RBE value calculated from the $01 \mathrm{HNG}$ cells data were not found in agreement with the classical RBE-LET. This discrepancy was not surprising because the yield of residual DSB was found higher than that of radioresistant controls (Fig. 6) and the SF2 of this cell line was found much lower $(25 \% \pm 2 \%$ vs $68 \% \pm 4 \%$, respectively). These numerical values strongly influence on the RBE calculations. Similarly, the RBE values calculated from the ATM-mutated AT5BI cell line were found invariable because the maximal hypersensitivity level is already reached with X-rays in these cell lines. It is noteworthy that we aimed to elucidate the wellknown RBE-LET relationship that was established from radioresistant cell lines. Hence, we did not apply the highLET radiation to cells from patients who already showed radiosensitivity with X-rays. Altogether, these data show that the ATM nucleo-shuttling model might help to predict the RBE-LET curves for any human cell line regardless of its radiosensitivity.

\section{Parallel between high/low-LET differences and individual radiation responses}

From the ATM nucleo-shuttling model, a classification of radiosensitivity in 3 groups was proposed ${ }^{22,34}$ :

- Group I: fast ATM nucleo-shuttling; complete DSB repair, radioresistance, and low cancer risk

- Group II: delayed ATM nucleo-shuttling; incomplete DSB repair, moderate radiosensitivity, and high cancer risk; neurofibromatosis; and Huntington disease are representative of genetic syndromes with group II radiosensitivity $^{16}$

- Group III: gross DSB repair defect regardless of the rate of ATM nucleo-shuttling, hyper-radiosensitivity, and high cancer risk with 2 subgroups: subgroup IIIa (delayed ATM nucleo-shuttling; eg, ataxia telangiectasia $\left[A T M^{-1}\right.$ mutations]); and subgroup IIIb (normal ATM nucleo-
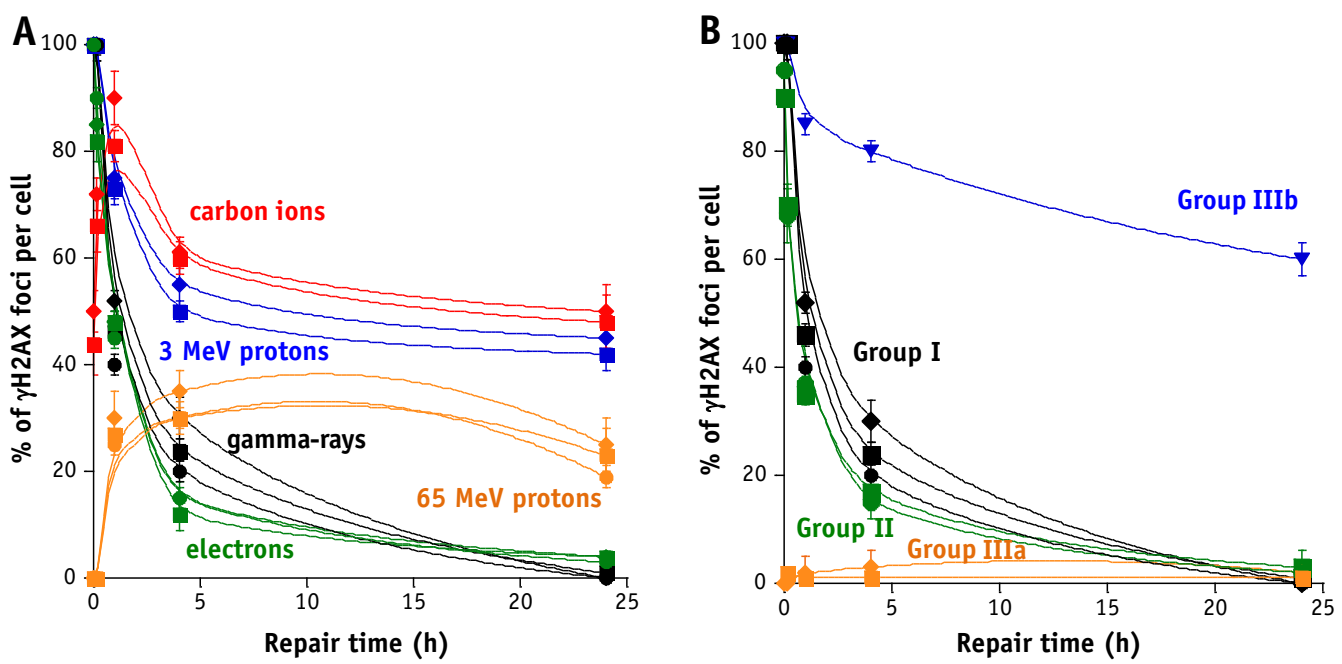

Fig. 6. Analogies in the response of radioresistant cells to high-LET particles and the response of radiosensitive cells to low-LET radiation. (A) The $\gamma \mathrm{H} 2 \mathrm{AX}$ foci data shown in Fig. 1 and expressed as a percentage are reproduced. (B) $\gamma \mathrm{H} 2 \mathrm{AX}$ foci kinetics data, also expressed as percentage, and obtained from cells representative of the 3 groups of radiosensitivity defined in the text and irradiated with $\gamma$-rays: group I, the radioresistant 149BR and 1BR3 fibroblasts; group II, the moderately radiosensitive $01 \mathrm{HNG}$ and $06 \mathrm{CLB}$ fibroblasts ${ }^{23}$; group IIIa, the hyper-radiosensitive ATM-mutated AT5BI fibroblasts; group IIIb, the hyper-radiosensitive LIG4-mutated 180BR fibroblasts. All these cell lines were characterized elsewhere. ${ }^{23}$ Each plot represents the mean \pm standard error of the mean of triplicates. 
shuttling; eg, the LIG4 syndrome $\left[L I G 4^{-/-}\right.$ mutations]) $)^{12,34}$

Interestingly, a number of similarities was observed in the shape of DSB repair kinetics between the exposure of radioresistant cells to high-LET particles and the exposure of radiosensitive cells to low-LET radiation (Fig. 6):

- The $\gamma \mathrm{H} 2 \mathrm{AX}$ kinetics of LIG4-mutated cell lines (group $\mathrm{IIIb}$ ) is similar to that obtained with $3-\mathrm{MeV}$ protons, reflecting a gross NHEJ-dependent DSB repair defect but a normal DSB recognition.

- The $\gamma \mathrm{H} 2 \mathrm{AX}$ kinetics of ATM-mutated cell lines (group IIIa) is similar to that obtained with $65-\mathrm{MeV}$ protons, reflecting a lack of DSB recognition combined with a gross NHEJ-dependent DSB repair defect.

- The $\gamma \mathrm{H} 2 \mathrm{AX}$ kinetics of group II cell lines (moderate radiosensitivity) is similar to that obtained with electrons, reflecting moderate impairments of DSB recognition and repair.

- The $\gamma \mathrm{H} 2 \mathrm{AX}$ kinetics obtained with carbon ions might correspond to intermediate case of group II (upper level) and group III, but we did not encounter such a case in our collection of human radiosensitive fibroblasts (data not shown).

\section{Conclusion}

All the repair data kinetics shown here obey the mathematical constraints detailed by Bodgi et al, ${ }^{34}$ the requirements linked to the ATM nucleo-shuttling model ${ }^{14}$ and suggest that our assumptions are consistent with the radiobiological effects linked to high-LET particles and low-LET radiation in quiescent cells. Further theoretical and experimental investigation is needed to better link physical and biological data and to develop reliable algorithms to predict the radiation response with a given LET and a given individual radiosensitivity.

\section{References}

1. Goodhead DT. The initial physical damage produced by ionizing radiations. Int J Radiat Biol 1989;56:623-634.

2. Goodhead DT. Spatial and temporal distribution of energy. Health Phys 1988:55:231-240.

3. Goodhead DT, Nikjoo H. Track structure analysis of ultrasoft x-rays compared with high- and low-let radiations. Int J Radiat Biol 1989;55: 513-529.

4. Goodhead DT, Thacker J, Cox R. Weiss lecture. Effects of radiations of different qualities on cells: Molecular mechanisms of damage and repair. Int J Radiat Biol 1993;63:543-556.

5. Ward JF. DNA damage produced by ionizing radiation in mammalian cells: Identities, mechanisms of formation, and reparability. Prog Nucleic Acid Res Mol Biol 1988;35:95-125.
6. Foray N, Charvet AM, Duchemin D, et al. The repair rate of radiationinduced DNA damage: A stochastic interpretation based on the gamma function. $J$ Theor Biol 2005;236:448-458.

7. Foray N, Monrocq C, Marples B, et al. Repair of radiation-induced DNA double-strand breaks in human fibroblasts is consistent with a continuous spectrum of repair probability. Int J Radiat Biol 1998;74:551-560.

8. Steel G. Basic Clinical Radiobiology. Arnold Publishers; 1993.

9. Li Y, Qian H, Wang H, et al. A stochastic model of DNA fragments rejoining. PloS One 2012;7:e44293.

10. Foray N, Bourguignon M, Hamada N. Individual response to ionizing radiation. Mutat Res Rev 2016;770:369-386.

11. Jeggo PA, Lobrich M. DNA double-strand breaks: Their cellular and clinical impact? Oncogene 2007;26:7717-7719.

12. Granzotto A, Benadjaoud MA, Vogin G, et al. Influence of nucleoshuttling of the ATM protein in the healthy tissues response to radiation therapy: Toward a molecular classification of human radiosensitivity. Int J Radiat Oncol Biol Phys 2016;94:450-460.

13. Belkacemi Y, Colson-Durand L, Granzotto A, et al. The Henri Mondor procedure of morbidity and mortality review meetings: Prospective registration of clinical, dosimetric, and individual radiosensitivity data of patients with severe radiation toxicity. Int J Radiat Oncol Biol Phys 2016:96:629-636.

14. Bodgi L, Foray N. The nucleo-shuttling of the ATM protein as a basis for a novel theory of radiation response: Resolution of the linearquadratic model. Int J Radiat Biol 2016;92:117-131.

15. Ferlazzo ML, Bach-Tobdji MKE, Djerad A, et al. Radiobiological characterization of tuberous sclerosis: A delay in the nucleo-shuttling of ATM may be responsible for radiosensitivity. Mol Neurobiol 2017; 55:4973-4983.

16. Ferlazzo ML, Sonzogni L, Granzotto A, et al. Mutations of the Huntington's disease protein impact on the ATM-dependent signaling and repair pathways of the radiation-induced DNA double-strand breaks: Corrective effect of statins and bisphosphonates. Mol Neurobiol 2014;49:1200-1211.

17. Granzotto A, Bencokova Z, Vogin G, et al. DNA double-strand breaks repair and signaling of human gliomas and normal brain cells in response to radiation: Potential impact of the ATM- and BRCA1-dependent pathways. In: Abujamra AL, editor. Brain Tumors. Book 3. Rijeka, Croatia. Intechweb; 2011

18. Pereira S, Bodgi L, Duclos M, et al. Fast and binary assay for predicting radiosensitivity based on the nucleoshuttling of ATM protein: Development, validation and performances. Int J Radiat Oncol Biol Phys 2018;100:353-360.

19. Baretic D, Pollard HK, Fischer DI, et al. Structures of closed and open conformations of dimeric human ATM. Sci Adv 2017;3:e1700933.

20. Paull TT. Mechanisms of ATM activation. Ann Rev Biochem 2015;84: 711-738.

21. Foray N, Priestley A, Alsbeih G, et al. Hypersensitivity of ataxia telangiectasia fibroblasts to ionizing radiation is associated with a repair deficiency of DNA double-strand breaks. Int J Radiat Biol 1997; 72:271-283.

22. Joubert A, Zimmerman KM, Bencokova Z, et al. DNA double-strand break repair defects in syndromes associated with acute radiation response: At least 2 different assays to predict intrinsic radiosensitivity? Int J Radiat Biol 2008;84:1-19.

23. Varela I, Pereira S, Ugalde AP, et al. Combined treatment with statins and aminobisphosphonates extends longevity in a mouse model of human premature aging. Nature Med 2008;14:767-772.

24. Foray N, Marot D, Gabriel A, et al. A subset of ATM- and ATRdependent phosphorylation events requires the brcal protein. EMBO $J$ 2003;22:2860-2871.

25. Foray N, Fertil B, Alsbeih MG, et al. Dose-rate effect on radiationinduced DNA double-strand breaks in the human fibroblast hf19 cell line. Int J Radiat Biol 1996;69:241-249.

26. Joubert A, Biston MC, Boudou C, et al. Irradiation in presence of iodinated contrast agent results in radiosensitization of endothelial cells: Consequences for computed tomography therapy. Int J Radiat Oncol Biol Phys 2005;62:1486-1496. 
27. Angellier G, Gautier M, Herault J. Radiochromic ebt2 film dosimetry for low-energy protontherapy. Med Phys 2011;38:6171-6177.

28. Bettega D, Calzolari P, Chauvel P, et al. Radiobiological studies on the $65 \mathrm{MeV}$ therapeutic proton beam at nice using human tumour cells. Int J Radiat Biol 2000;76:1297-1303.

29. Courdi A, Caujolle JP, Grange JD, et al. Results of proton therapy of uveal melanomas treated in nice. Int J Radiat Oncol Biol Phys 1999; 45:5-11.

30. Beuve M, Alphonse G, Maalouf M, et al. Radiobiologic parameters and local effect model predictions for head and neck squamous cell carcinomas exposed to high linear energy transfer ions. Int J Radiat Oncol Biol Phys 2008;71:635-642.

31. Hanot M, Boivin A, Malesys C, et al. Glutathione depletion and carbon ion radiation potentiate clustered DNA lesions, cell death and prevent chromosomal changes in cancer cells progeny. PloS One 2012; 7:e44367.

32. Maalouf M, Alphonse G, Colliaux A, et al. Different mechanisms of cell death in radiosensitive and radioresistant p53 mutated head and neck squamous cell carcinoma cell lines exposed to carbon ions and xrays. Int J Radiat Oncol Biol Phys 2009;74:200-209.
33. Foray N, Arlett CF, Malaise EP. Radiation-induced DNA doublestrand breaks and the radiosensitivity of human cells: A closer look. Biochimie 1997;79:567-575.

34. Bodgi L, Granzotto A, Devic C, et al. A single formula to describe radiation-induced protein relocalization: Towards a mathematical definition of individual radiosensitivity. J Theor Biol 2013;333:135145.

35. Foray N, Badie C, Alsbeih G, et al. A new model describing the curves for repair of both DNA double-strand breaks and chromosome damage. Radiat Res 1996;146:53-60.

36. Sorensen BS, Overgaard J, Bassler N. In vitro RBE-LET dependence for multiple particle types. Acta Oncol 2011;50:757-762.

37. Tommasino F, Durante M. Proton radiobiology. Cancers 2015;7:353381.

38. Kuhne M, Riballo E, Rief N, et al. A double-strand break repair defect in ATM-deficient cells contributes to radiosensitivity. Cancer Res 2004;64:500-508.

39. Stiff T, O'Driscoll M, Rief N, et al. ATM and DNA-pk function redundantly to phosphorylate $\mathrm{h} 2 \mathrm{ax}$ after exposure to ionizing radiation. Cancer Rest 2004;64:2390-2396. 